Journal for ImmunoTherapy of Cancer

\section{Improved prognosis and evidence of enhanced immunogenicity in tumor and circulation of high-risk melanoma patients with unknown primary}

To cite: Tarhini AA, Lee SJ, Tan A-C, et al. Improved prognosis and evidence of enhanced immunogenicity in tumor and circulation of highrisk melanoma patients with unknown primary. Journal for ImmunoTherapy of Cancer 2022;10:e004310. doi:10.1136/ jitc-2021-004310

- Additional supplemental material is published online only. To view, please visit the journal online (http://dx.doi.org/10. 1136/jitc-2021-004310).

Presented at the Annual Meeting of the American Society of clinical Oncology, Chicago, IL, May 28, 2020-May 30, 2020 and the Society for Immunotherapy of Cancer's (SITC) 36th Annual Meeting, Washington, DC, November 10 , 2021-14, November, 2021.

Accepted 28 December 2021

Check for updates

(C) Author(s) (or their employer(s)) 2022. Re-use permitted under CC BY-NC. No commercial re-use. See rights and permissions. Published by BMJ.

For numbered affiliations see end of article.

Correspondence to

Professor Ahmad A Tarhini; ahmad.tarhini@moffitt.org

\section{ABSTRACT}

Background Melanoma of unknown primary (MUP) represents a poorly understood group of patients both clinically and immunologically. We investigated differences in prognosis and candidate immune biomarkers in patients with unknown compared with those with known primary melanoma enrolled in the E1609 adjuvant trial that tested ipilimumab at 3 and $10 \mathrm{mg} / \mathrm{kg}$ vs high-dose interferon-alfa (HDI).

Patients and methods MUP status was defined as initial presentation with cutaneous, nodal or distant metastasis without a known primary. Relapse-free survival (RFS) and overall survival (OS) rates were estimated by the KaplanMeier method. Stratified (by stage) log-rank test was used to compare RFS and OS by primary tumor status. Gene expression profiling (GEP) was performed on the tumor biopsies of a subset of patients. Similarly, peripheral blood samples were tested for candidate soluble and cellular immune biomarkers.

Results MUP cases represented $12.8 \%$ of the total population ( $\mathrm{N}=1699$ ) including $11.7 \%$ on the ipilimumab arms and $14.7 \%$ on the HDI arm. Stratifying by stage, RFS $(p=0.001)$ and overall survival $(0 S)(p=0.009)$ showed outcomes significantly better for patients with unknown primary. The primary tumor status remained prognostically significant after adjusting for treatment and stage in multivariate Cox proportional hazards models. Including only ipilimumab-treated patients, RFS ( $p=0.005)$ and OS $(p=0.023)$ were significantly better in favor of those with unknown primary. Among patients with GEP data $(n=718$; 102 MUP, 616 known), GEP identified pathways and genes related to autoimmunity, inflammation, immune cell infiltration and immune activation that were significantly enriched in the MUP tumors compared with known primaries. Further investigation into infiltrating immune cell types estimated significant enrichment with CD8 +and CD4+T cells, B cells and NK cells as well as significantly higher major histocompatibility complex (MHC)-I and MHC-Il scores in MUP compared with known primary. Among patients tested for circulating biomarkers $(n=321$; 66 unknown and 255 known), patients with MUP had significantly higher circulating levels of IL-2R $(p=0.04)$. Conclusion Patients with MUP and high-risk melanoma had significantly better prognosis and evidence of significantly enhanced immune activation within the TME and the circulation, supporting the designation of MUP as a distinct prognostic marker in patients with high-risk melanoma.

\section{INTRODUCTION}

The incidence of melanoma continues to rise annually with 106110 new invasive melanoma cases and 7180 deaths estimated in the USA in the year 2021. ${ }^{1}$ This increase in incidence similarly applies to high-risk melanoma including patients with resectable regional nodal (stage III) or distant (stage IV) metastatic disease, who can be rendered disease free surgically, but continue to be at a high risk for disease relapse and death from melanoma that rises with stage. ${ }^{2}{ }^{3}$ Unknown primary melanoma represents a significant proportion of the disease, and unknown primary is typically attributed to the phenomenon of complete regression at the primary melanoma site. ${ }^{4}$ The American Joint Committee on Cancer (AJCG) staging system divides cutaneous melanoma into four stages. ${ }^{4}$ Stages I and II include patients where the detectable primary melanoma is confined to the skin and where prognosis is primarily defined by the depth of the tumor and its ulceration status. Stage III includes patients with regional nodal metastases where the primary tumor depth and ulceration status continue to impact prognosis and staging. Stage IV is defined by the spread of metastasis into distant sites. When it comes to melanoma of unknown primary (MUP), there are limited data about prognosis and how these patients should be optimally staged. ${ }^{4}$ Retrospective single institutional analyses have suggested improved survival compared with similarly staged patients with known primary, 
based on the sites of metastases. ${ }^{5-7}$ However, the AJCC staging system recommends that patients with MUP (T0) who present with lymph node metastases be assigned to the corresponding $\mathrm{N}$ category based on the number of metastatic lymph nodes and the presence or absence of intransit metastases while acknowledging that the proposed staging should be used until additional data are available. ${ }^{4}$ Clearly, additional data are needed in order to better define the prognosis of this patient population and better understand the underlying biology and immunogenicity of unknown primary melanomas. Such data may have implications related to staging and the design of future adjuvant trials where unknown primary status could be accounted for as a stratification factor.

The North American Intergroup trial E1609 tested ipilimumab at $3 \mathrm{mg} / \mathrm{kg}$ (ipi3) or $10 \mathrm{mg} / \mathrm{kg}$ (ipi10) vs high-dose interferon- $\alpha$ (HDI) and demonstrated significant overall survival (OS) benefits with ipi3 compared with HDI and less toxicity compared with ipi10. ${ }^{8}$ This trial is the second largest adjuvant trial reported to date in melanoma with participation from 1673 patients. ${ }^{8}$ E1609 allowed the enrolment of melanoma patients with unknown primary that were randomized across the three arms of the study. We observed that a significant proportion of E1609's adult patients had an unknown primary melanoma and have, therefore, conducted an in-depth analysis to better understand the clinical and prognostic features of this patient population as compared with similarly staged patients with a known primary. Furthermore, we conducted tumor tissue and blood based immune monitoring studies in consenting patients with available biospecimens. This report presents the findings from our clinical and laboratory immune monitoring analyses.

\section{Patients and methods}

Patients

The phase III E1609 randomized patients with melanoma of cutaneous or unknown primary origin that was histologically confirmed and had AJCC seventh edition stages IIIB, IIIC or IV (M1a or M1b) and were rendered disease free surgically within 12 weeks of registration on the trial. ${ }^{8}$ An Eastern Cooperative Oncology Group (ECOG) performance status of 0 or 1 and safety laboratory test criteria were mandated at screening. Patients were excluded if they had a history of autoimmune disorders or conditions of immunosuppression that necessitated the use of systemic corticosteroids or other immunosuppressants.E

\section{Trial design and treatments}

E1609 was an open-label phase III trial where patients were randomized to adjuvant therapy with either ipi3, ipi10 or HDI. Patients were stratified by stage (AJCC seventh edition IIIB, IIIC, M1a, M1b). ${ }^{8}$ Details of the treatment regimens, randomization procedures, clinical trial endpoint points and trial oversight were previously published. ${ }^{8}$ Patient disposition is described in the consort diagram included in online supplemental figure S1.

\section{Methods and statistical analysis}

Unknown primary melanoma status was defined as initial presentation with cutaneous, nodal or lung metastasis that was completely surgically resected without a history of known primary melanoma as registered on the study. All pathology reports were required to be submitted for each surgical intervention related to melanoma management and centrally reviewed by the study's principal investigator. Central histology review was conducted on a subset of cases by the study's pathology cochair (UR). Individual records and pathology reports were reviewed by study chair (AAT) in order to verify patient designated AJCC stage working closely with the study's pathology cochair (UR) and the study team. Patient distribution by the initial site of metastasis was analyzed. Further, we evaluated the risk of relapse and death by the primary tumor status and by the AJCG stage group. Five-year relapse-free survival (RFS) and overall survival (OS) rates and 95\% CIs were estimated by the Kaplan-Meier method. Stratified (by stage) log-rank test was used to compare RFS and OS by primary tumor status in the overall study population and among the different study arms. Two-sided $p$ values were reported.

\section{Gene expression profiling}

Gene expression profiling (GEP) was performed on the tumor biopsies of 718 (102 unknown, 616 known primary) melanoma patients. Only metastatic tumors were included that were resected to render patients' disease free prior to clinical trial enrolment. Microdissection of FFPE tumor specimens was performed manually using an inverted microscope (Nikon Eclipse TE200) as needed to obtain a minimum of $90 \%$ tumor cells for RNA purification. Dissection involved scraping cells from unstained sections of $5 \mu \mathrm{m}$ thickness on slides aligned in register with serially cut H\&E-stained specimens including tumor domains demarcated by a surgical pathologist (ADK). RNA purification was performed using the Qiagen miRNeasy FFPE Kit and protocol (Qiagen, Valencia, California, USA) with isolated RNA suspended in nuclease-free water. Inclusion in subsequent in vitro amplification assays was determined both by spectrophotometric absorption ratio (260/280>1.8 (NanoDrop, Wilmington, Delaware, USA)) and RIN values (RNA Integrity Index) determined via microchip electrophoretic analysis (Agilent Bioanalyzer 2100, Agilent Technologies, Santa Clara, California, USA). We previously established that RIN values ranging from 5.0 to 8.0 in RNA from FFPE specimens can undergo successful in vitro transcription and amplification using a multiplex primer approach. Amplification was performed using the NuGen whole transcription method comprizing the Ovation FFPE WTA assay (NuGEN, San Carlos, California, USA) employing random and 3' primers to eliminate amplification bias beginning with $100 \mathrm{ng}$ total RNA. Confirmation of cDNA diversity was obtained using the Bioanalyzer 2100 to generate an electrophoretogram for each amplification reaction regarding sample yield, integrity and size diversity compared with a laboratory human 
RNA standard and a Universal Human Reference RNA (Stratagene, La Jolla, California, USA). A $5 \mu \mathrm{g}$ of purified cDNA was incubated with fragmentation buffer (NuGEN, San Carlos, CA) at $37^{\circ} \mathrm{C}$ for $30 \mathrm{~min}$, then $95^{\circ} \mathrm{C}$ for $2 \mathrm{~min}$. All cDNA samples underwent hybridization on Affymetrix GeneChip HG U133A 2.0 arrays which contain overlapping probe sets for transcripts comprehensively representing the functionally characterized human genome. Briefly, fragmented cDNAs were mixed in a hybridization cocktail with water to a final volume of $220 \mu \mathrm{L} .130 \mu \mathrm{L}$ of hybridization cocktail is hybridized on each array at $45^{\circ} \mathrm{C}$ for 18 hours. The arrays were then washed and stained with streptavidin-phycoerythrin in a GeneChip Fluidics Station 450 (Affymetrix) and scanned using a GeneChip Scanner 3000 (Affymetrix). Raw microarray data sets were normalized by using the Robust Multi-array Average (RMA) method using Affymetrix Power Tools (APT) as previously published. ${ }^{9}$ Multiple probe sets representing the same genes were collapsed by using the probe with maximum gene expression. Gene set enrichment analysis (GSEA) was performed by comparing the unknown and known primary tumor samples. ${ }^{10}$ For this purpose, KEGG pathways gene sets were obtained from MSigDB (PMID: 21546393) to interrogate the enrichment of pathways in the unknown versus known primary samples. ${ }^{11}$ In order to further to deconvolute the cell types in the bulk transcriptomics, we used gene sets obtained from CIBERSORT $^{12} 13$ and TIMEx ${ }^{14}$ in comparing the unknown vs known primary samples. Gene sets with a false discovery rate $q$-value $<0.1$ were deemed as significant. Major histocompatibility complex (MHC)-I and MHC-II scores were generated from bulk gene expression profiles by comparing unknown primary tumors and known primaries. For each sample, the scores were computed by averaging the standardized z-score of 6 MHC-I genes (HLA-A, HLA-B, HLA-C, B2M, TAP1, TAP2) and 13 MHC-II genes (HLA-DMA, HLA-DMB, HLA-DOA, HLA-DOB, HLADPA1, HLA-DPB1, HLA-DQA1, HLA-DQA2, HLA-DQB1, HLA-DQB2, HLA-DRA, HLA-DRB1, HLA-DRB5) as previously described. ${ }^{15}$ Mann-Whitney $\mathrm{U}$ test was performed between the two groups and $\mathrm{p}<0.05$ was deemed as statistically significant.

Similarly, peripheral blood mononuclear cells (serum and PBMC) samples collected at baseline (prior to initiating systemic therapy) were tested for soluble and cellular immune biomarkers in a subset of patients $(\mathrm{N}=321 ; 66$ unknown and 255 known primary).

\section{Blood processing}

Red top vacutainer tubes (BD, no anticoagulant) were used for serum collection and all samples were processed within 24 hours of collection (samples received before 17:00 hour were processed on receipt, those arriving after 17:00 hour were processed the following morning). Serum samples were centrifuged at $2500 \mathrm{rpm}$ for $10 \mathrm{~min}$ at $4^{\circ} \mathrm{C}$ according to laboratory standard operating procedures (SOPs) and single use aliquots of each patient's sera were then stored at $-80^{\circ} \mathrm{C}$. The laboratory freezers were monitored continuously for any temperature fluctuations and maintained the samples at $-80^{\circ} \mathrm{C}$.

\section{Multiplex serum cytokine analysis}

Twenty-one serum cytokines were selected for analysis based on function. These included Th1 type cytokines (IL-12p70, IL-17, IL-2, IP-10), proinflammatory (IL-1 $\alpha$, IL-1 $\beta$, IL-6, TNF- $\alpha$, TNF-RII, IL-2R, IL-8, C reactive Protein (CRP), IL-17, IFN- $\alpha$ ), immunoregulatory (TGF- $\alpha$, IL-10, TIMP1), growth factor (VEGF-A), and other/chemokines (CCL3/MIP-1 $\alpha$, CCL4/MIP-1 $\beta$, CXCL9/MIG, CXCL11/ I-TAC). The xMAP Luminex serum assay for these cytokines was performed according to the manufacturer's protocol (BioSource International (Camarillo, California, USA) as previously described,${ }^{16}$ and laboratory SOPs, and analyzed on the Bio-Plex suspension array system (BioRad Laboratories, Hercules, California, USA). Experimental data were analyzed using five-parametric curve fitting and assay controls included kit standards and multiplex QC controls (R\&D Systems). Interassay variabilities for individual cytokines were $1.0 \%-9.8 \%$ and intra-assay variabilities were $3.6 \%-12.6 \%$ (information provided by Biosource International and validation performed in our laboratory). CRP was run singly as it requires different dilutions.

\section{Multicolor flow cytometry}

Multicolor flow cytometry was used to compare cell subset phenotypes on thawed patient PBMC, with healthy donor controls, run according to laboratory SOPs. Regulatory $\mathrm{T}$ cells (Treg) were defined as CD4 +CD25+FOXP3+or CD4+CD25hi+CD39+cells, to incorporate the candidate functional marker CD39 as previously described. ${ }^{17}$ Myeloid-derived-suppressor cells were defined as cells expressing Lin-neg/HLA-DR-/CD33+/CD11b+in either a 'lymphocyte' (small FSCxSSC) gate, or in a 'monocyte' (larger FSCxSSC) gate, and as HLA-DR+/lo CD14 + cells in a large gate. We also tested the frequencies of CD4 +and CD8+T cells specific to shared tumor-associated antigens (Gp-100, MART-1, NY-ESO-1) using overlapping peptide libraries (15-mer peptides overlapping by 4 ) and a short (4-5 hours) in vitro culture to identify activated (CD69+) and cytokine producing (intracellular IFN $\gamma+$ ) $\mathrm{T}$ cells. Detailed methods were described previously. ${ }^{17}$

\section{RESULTS}

Patient characteristics and treatment details were previously published. ${ }^{8}$ Unknown primary melanoma cases represented $12.8 \%$ of the total study population included in this analysis (214/1669) including 11.7\% (121/1034) on the ipilimumab arms and $14.6 \%(93 / 635)$ on the HDI arm. Site of metastasis at initial presentation among the unknown primary cases included cutaneous and nodal (97\%; 202/208) and lung (3\%; 6/208). Among the overall E1609 study population, the proportion of patients with unknown primary was $10.5 \%$ (92/879) for 

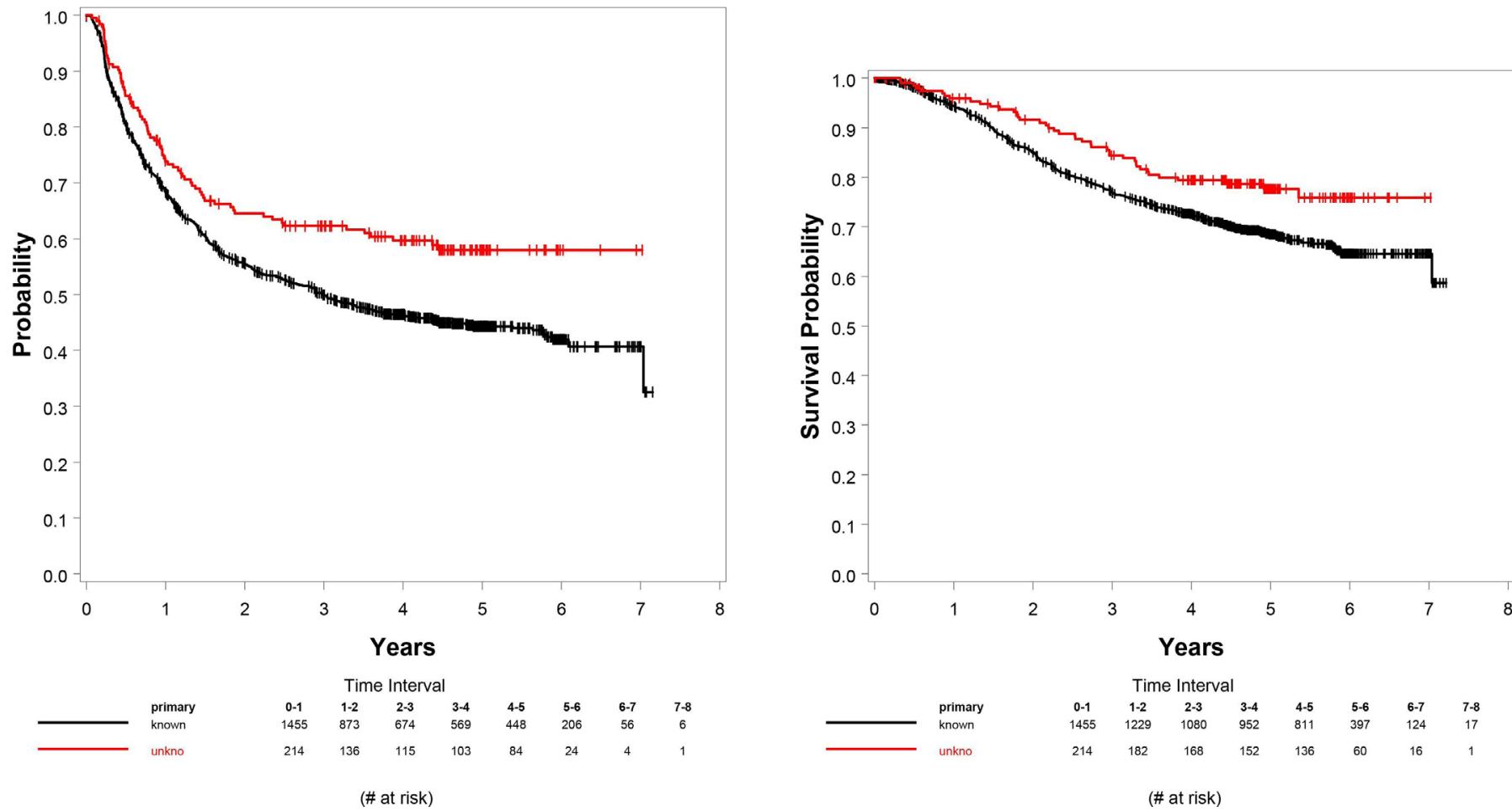

Figure 1 Kaplan-Meier curves for relapse-free survival (RFS) (A) and overall survival (OS) (B) for patients with unknown primary melanoma compared with those with known primary. Stratifying by treatment and stage, RFS $(p=0.001)$ and OS $(p=0.008)$ were significantly better for patients with unknown primary tumor compared with known primary.

IIIB, $11.6 \%$ (77/662) for IIIC, $42.2 \%$ (38/90) for M1a and $18.4 \%$ (7/38) for M1b across all treatment arms.

Stratifying by treatment and stage, RFS $(p=0.001)$ and OS $(p=0.008)$ were significantly better for patients with unknown primary tumor compared with known primary. Figure 1A,B shows the Kaplan-Meier curves for RFS and OS, respectively for all patients. Five-year RFS and OS rates by primary tumor status and stage group among all patients enrolled on the study are summarized in online supplemental table S1. Improvements in RFS and OS were consistently in favor of the unknown primary status across the stage groups (IIIB, IIIC and M1a/M1b). Furthermore, the primary tumor status remained significant after adjusting for treatment and stage in multivariate Cox proportional hazards models.

Including only ipi3 and ipi10 patients, RFS ( $p=0.005)$ and OS $(p=0.024)$ were consistently significantly better in favor of patients with unknown primary status. Figure 2A,B shows the Kaplan-Meier curves for RFS and OS, respectively for ipilimumab-treated patients. Five-year RFS and OS rates by primary tumor status and stage group among patients treated with ipilimumab (ipi3 and ipi10 arms) are summarized in online supplemental table S1.

Including only HDI patients, RFS $(\mathrm{p}=0.055)$ and OS $(\mathrm{p}=0.129)$ were marginally better in favor of the unknown primary status. Figure 3A,B shows the Kaplan-Meier curves for RFS and OS, respectively for HDI-treated patients. Five-year RFS and OS rates by primary tumor status and stage group among patients treated with HDI are summarized in online supplemental table S1.

GSEA identified pathways and genes related to autoimmunity, inflammation, immune cell infiltration and immune activation that were significantly enriched in the unknown primary tumors compared with known primaries (table 1). TIMEx revealed that unknown primary tumors were enriched with immune cell types infiltrated in the bulk transcriptomics, especially CD8 +and CD4+T cells, B cells and NK cells (TIMEx, table 2). Similar results were also identified by interrogating the CIBERSORT signatures (CIBERSORT, online supplemental table S2). On the other hand, known primaries were enriched with stromal fibroblasts and myofibroblasts (table 2).

MHC-I and MHC-II scores were computed from bulk gene expression profiles. MUP tumors had significantly higher MHC-I $(p=0.004)$ and MHC-II $(p=0.023)$ scores as compared with known primary tumors (figure 4).

Among the subset of patients tested for circulating biomarkers, patients with unknown primary had significantly higher levels of IL-2R than those with known primary $(\mathrm{p}=0.04)$ as shown in figure 5 .

\section{DISCUSSION}

MUP appears to represent a unique patient population with improved prognosis compared with similarly staged melanoma patients with known primary. This study was 


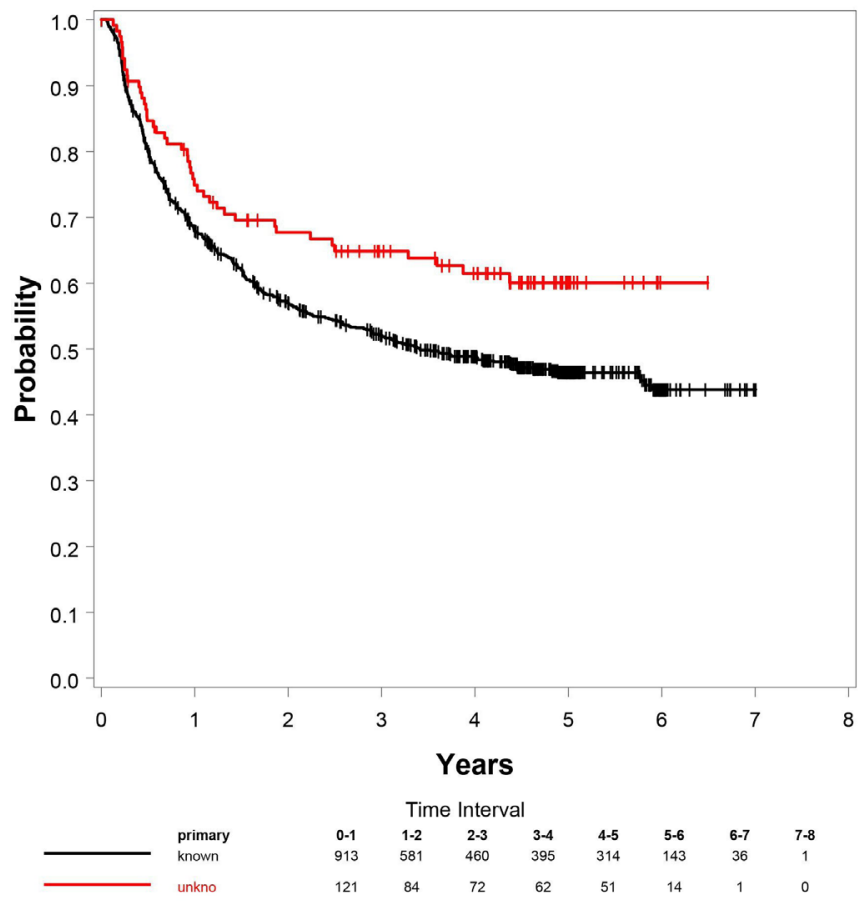

(\# at risk)

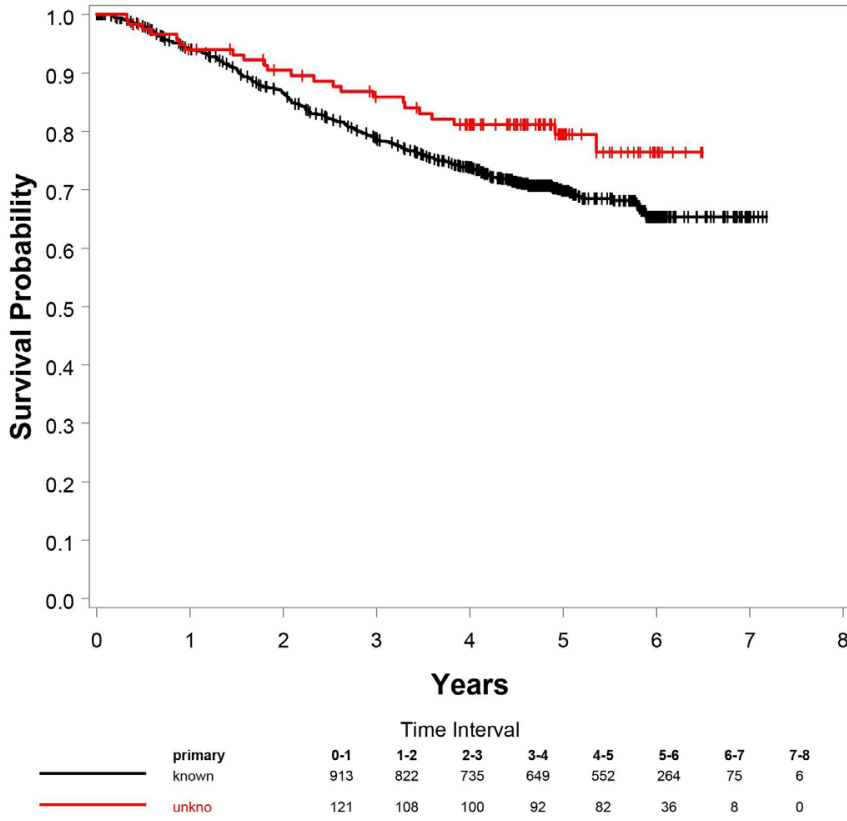

(\# at risk)

Figure 2 Kaplan-Meier curves for relapse-free survival (RFS) (A) and overall survival (OS) (B) for ipilimumab-treated patients with unknown primary melanoma compared with those with known primary. RFS $(p=0.005)$ and OS $(p=0.024)$ were consistently significantly better in favor of the unknown primary status.

\section{A E1609 Recurrence-Free Survival in HDI Arm by Primary Status}
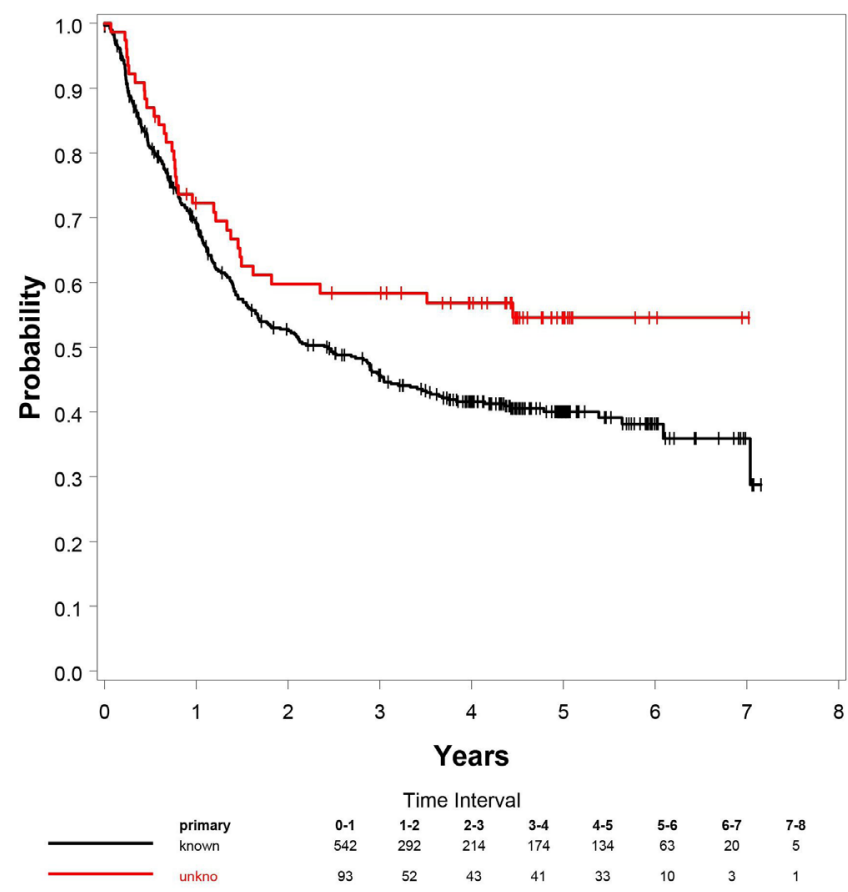

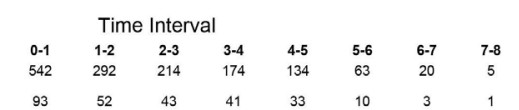

(\# at risk)

\section{B E1609 Overall Survival in HDI Arm by Primary Status}

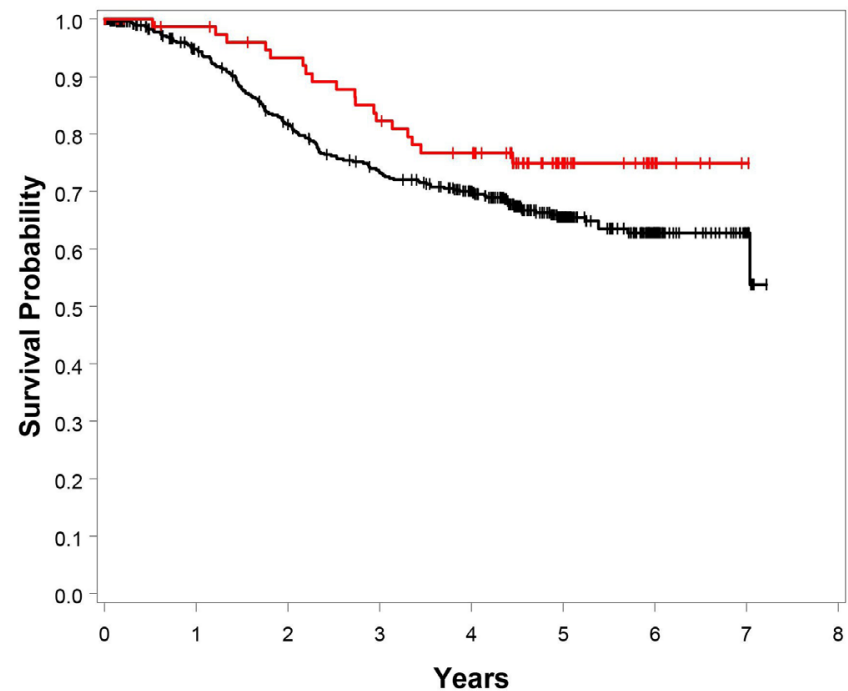

primary

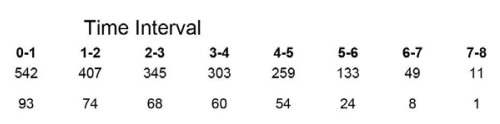

(\# at risk)

Figure 3 Kaplan-Meier curves for relapse free survival (RFS) (A) and overall survival (OS) (B) for high dose interferon-alfa (HDI)treated patients with unknown primary melanoma compared with those with known primary. $\operatorname{RFS}(p=0.055)$ and OS $(p=0.129)$ were marginally better in favor of the unknown primary status. 
Table 1 Immune related pathways found to be significantly enriched in unknown primary melanomas compared with known primary as computed by gene set enrichment analysis (using KEGG pathways gene sets) (NES: Normalized Enrichment Score)

\begin{tabular}{llll}
\hline KEGG pathways & NES & NOM p value & FDR q value \\
\hline Antigen processing and presentation & 2.28 & 0.0000 & 0.0000 \\
\hline Autoimmune thyroid disease & 2.25 & 0.0000 & 0.0000 \\
\hline Allograft rejection & 2.22 & 0.0000 & 0.0000 \\
\hline Systemic lupus erythematosus & 2.21 & 0.0000 & 0.0000 \\
\hline Intestinal immune network for iga production & 2.11 & 0.0000 & 0.0002 \\
\hline Graft versus host disease & 2.07 & 0.0000 & 0.0001 \\
\hline Leishmania infection & 1.98 & 0.0000 & 0.0007 \\
\hline Primary immunodeficiency & 1.96 & 0.0000 & 0.0011 \\
\hline Type I diabetes mellitus & 1.94 & 0.0000 & 0.0011 \\
\hline DNA Replication & 1.70 & 0.0097 & 0.0386 \\
\hline Toll like receptor signaling pathway & 1.69 & 0.0000 & 0.0364 \\
\hline Viral myocarditis & 1.69 & 0.0031 & 0.0342 \\
\hline Pantothenate and Coa Biosynthesis & 1.68 & 0.0203 & 0.0358 \\
\hline Prion diseases & 1.63 & 0.0084 & 0.0564 \\
\hline Natural killer cell mediated cytotoxicity & 1.63 & 0.0030 & 0.0531 \\
\hline Protein export & 1.62 & 0.0188 & 0.0529 \\
\hline Asthma & 1.59 & 0.0223 & 0.0633 \\
\hline Starch and sucrose metabolism & 1.57 & 0.0161 & 0.0776 \\
\hline Complement and coagulation cascades & 1.56 & 0.0142 & 0.0774 \\
\hline Cell adhesion molecules cams & 1.55 & 0.0031 & 0.0777 \\
\hline
\end{tabular}

FDR, false discovery rate; NOM $p$-val, Nominal $p$ value.

nested within the E1609 randomized controlled trial that enrolled patients with AJCC seventh edition stages IIIB, IIIC, M1a and M1b operable melanoma that remain at high risk for recurrence and death following surgical resection. The E1609 study allowed MUP as part of the eligibility criteria allowing for the enrolment of a relatively large sample size that represented $12.8 \%$ of the total study population. While MUP was not a stratification factor in E1609, MUP cases were similarly distributed between the ipilimumab arms and the HDI arm $(11.7 \%$ and $14.7 \%$, respectively). Although the study enrolled patients with resected lung metastases, these cases represented only 3\% of the MUP population and the overwhelming majority of MUP were cutaneous and nodal metastases. In terms of RFS and OS, patients with MUP had significantly better outcomes in the overall study population after adjusting for treatment and stage. The significant improvements in RFS and OS in favor of MUP persisted when the analysis included only ipilimumab treated cases, but were only marginally better when including HDI cases alone. This may be explained in terms of ipilimumab's superior adjuvant efficacy in melanoma especially when considering a more immunogenic TME of MUP, which may potentially be predictive of response to immune checkpoint inhibitors. ${ }^{18}$ These data support the hypothesis that MUP represents a distinct patient population with significantly better prognosis compared with similarly staged patients according to the AJCC staging system. To date, evidence in the literature to support this hypothesis has been limited to retrospective case series. ${ }^{19}$ A retrospective analysis of melanoma patients diagnosed between 1990 and 2001 who underwent surgical resection of regional lymph nodes compared two cohorts including patients with MUP and control patients with a median follow-up of 7.7 years. ${ }^{20}$ OS rates at 5 and 10 years were $55 \%$ and $44 \%$, respectively, for patients with MUP, compared with $42 \%$ and $32 \%$ for the control group with known primary $(\mathrm{p}=0.04)$. Moreover, in multivariate analyses, MUP was found to be a favorable prognostic factor for OS, with HR $0.61,95 \%$ CI $(0.42$ to $0.86 ; p=0.006)$ apart from therapy. Similarly, a retrospective study of a single institution melanoma database reported significantly higher overall survival rates after surgical resection for melanoma patients with lymphadenopathy from MUP. Five-year OS rates $55 \%$ compared with $44 \%$ in favor of MUP; $\mathrm{p}=0.00210{ }^{6}$ Another retrospective study also reported similar observations where patients with MUP had significantly better survival outcomes compared with those with a known primary. ${ }^{7}$ Interestingly, case series that performed molecular characterization of unknown primary cases have suggested a similar incidence of $B R A F$ and NRAS mutations to that expected in known cutaneous primaries. ${ }^{21}$ 
Table 2 Tumor immune microenvironment cell types estimated by TIMEx signatures found to be significantly enriched in unknown primary melanomas compared with known primary and vice versa as computed by GSEA (NES: Normalized Enrichment Score)

\begin{tabular}{llll}
\hline & NES & NOM p value & FDR q value \\
\hline Enriched in unknown primary tumors & & & \\
\hline IMMUNE_CD8TEX & 2.53 & 0.0000 & 0.0000 \\
\hline IMMUNE_B & 2.34 & 0.0000 & 0.0000 \\
\hline IMMUNE_NK & 2.22 & 0.0000 & 0.0000 \\
\hline IMMUNE_PLASMA & 2.21 & 0.0000 & 0.0000 \\
\hline IMMUNE_CD4TCONV & 2.17 & 0.0000 & 0.0000 \\
\hline IMMUNE_CD8T & 2.15 & 0.0000 & 0.0000 \\
\hline MINOR_CD8TCM & 2.13 & 0.0000 & 0.0000 \\
\hline MINOR_CD4TN & 2.11 & 0.0000 & 0.0000 \\
\hline IMMUNE_TMKI67 & 1.98 & 0.0000 \\
\hline MINOR_TH1 & 1.95 & 0.0000 & 0.0000 \\
\hline IMMUNE_DC & 1.94 & 0.0000 & 0.0000 \\
\hline MINOR_CDC2 & 1.91 & 0.0000 & 0.0001 \\
\hline MINOR_CD8TEFF & 1.90 & 0.0000 & 0.0001 \\
\hline MINOR_CD4TEFF & 1.89 & 0.0000 & 0.0001 \\
\hline MINOR_M1 & 1.87 & 0.0000 & 0.0001 \\
\hline IMMUNE_PDC & 1.82 & 0.0000 & 0.0004 \\
\hline IMMUNE_TREG & 1.82 & 0.0000 & 0.0003 \\
\hline MINOR_MONOCYTE & 1.51 & 0.0000 & 0.0168 \\
\hline MINOR_TH17 & 1.50 & 0.0079 & 0.0184 \\
\hline IMMUNE_NEUTROPHILS & 1.50 & 0.0361 & 0.0184 \\
\hline IMMUNE_ILC & 1.38 & 0.0162 & 0.0467 \\
\hline Enriched in known primaries & & 0.0666 & 0.0799 \\
\hline STROMAL_FIBROBLASTS & -1.51 & & 0.0484 \\
\hline STROMAL_MYOFIBROBLASTS & -1.48 & 0.0000 & 0.0000 \\
\hline FRR & & \\
\hline
\end{tabular}

FDR, false discovery rate; GSEA, gene set enrichment analysis.

In order to investigate the underlying molecular pathways in MUP that contribute to improved outcomes, we performed GSEA by comparing MUP tumors to known primaries in a subset of patients using gene expression profiles. For this purpose, we used KEGG pathway gene sets, a reference database for pathway mapping representing knowledge of molecular interaction, reaction and relation networks for metabolism, genetic information processing, environmental information processing, cellular processes, organismal systems, human diseases and drug development. ${ }^{22}$ Our GSEA findings supported evidence of enhanced expression of immune-related genes and the activation of immune related pathways in MUP. The top pathways identified via GSEA and associated molecules were notably immune related and highly statistically significantly associated with MUP tumors over those with known primary. Next, we used CIBERSORT and TIMEx in order to deconvolute the cell types in the bulk transcriptomics. ${ }^{12} 14$ TIMEx, our recently developed portal of tumor immune microenvironment cell type signatures collected from pan-cancer single cell RNA sequencing data sets, is a tumor immune microenvironment deconvolution method that emphasizes estimating infiltrating immune cell types. ${ }^{14}$ It includes 37 tumor immune microenvironment cell type signatures. Using TIMEx, the infiltrating immune cell types in the unknown primaries were enriched with $\mathrm{T}$ cells, B cells and NK cells. These findings were also validated by using CIBERSORT leukocytes gene signatures that includes 22 human hematopoietic cell phenotypes. Immune-related gene expression profiles were found to be prognostic of improved outcome in melanoma and other malignancies and potentially predictive of clinical benefit in patients treated with immune checkpoint inhibitors. ${ }^{18}$ We previously reported an immune related 22-gene expression signature detected in the tumors of patients treated with neoadjuvant ipilimumab that was significantly associated with clinical benefit. ${ }^{18}$ These genes constituted a proinflammatory gene expression profile of chemokines and other immune-related genes associated with a Type I 


\section{MHC-I Score}

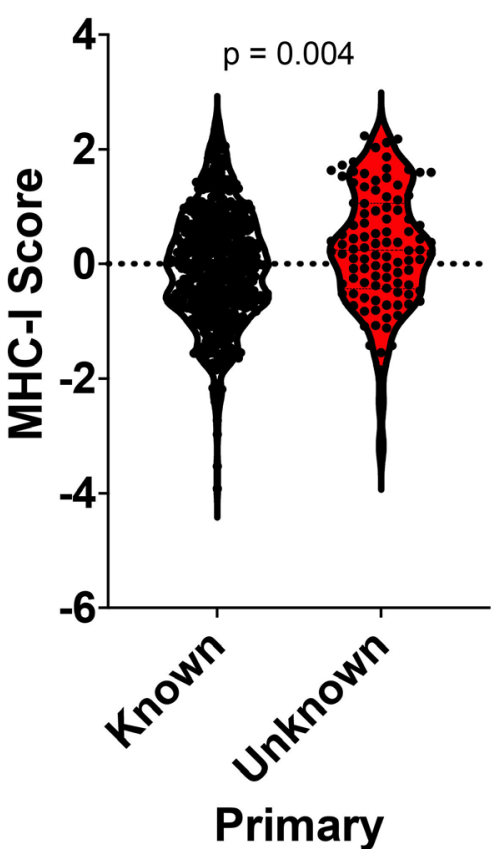

MHC-II Score

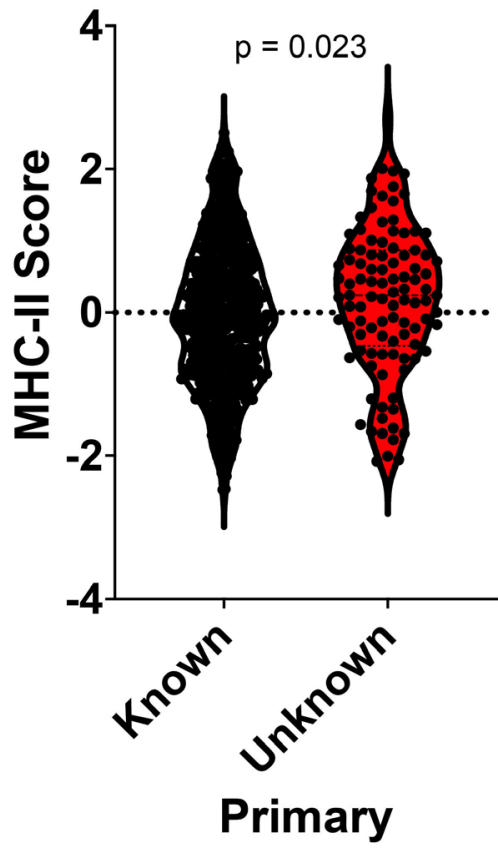

Figure 4 Major histocompatibility complex (MHC)-I and MHC-II scores were computed from bulk gene expression profiles by comparing unknown primary melanoma (MUP) tumors and known primaries. MUP had significantly higher MHC-I $(p=0.004)$ and MHC-II $(p=0.023)$ scores as compared with known primary.

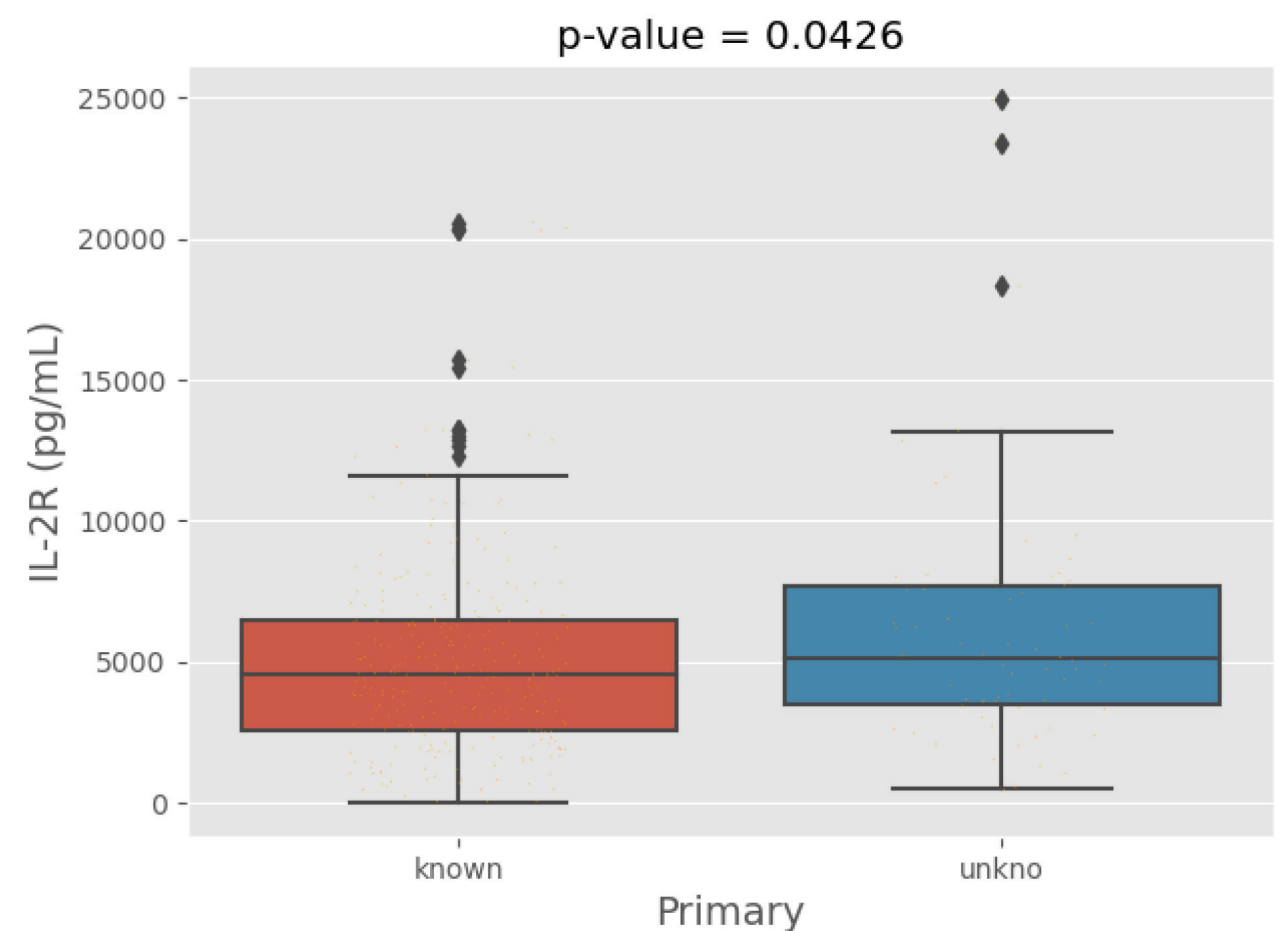

Mann-Whitney U test

Figure 5 Patients with unknown primary melanoma had higher serum levels of IL-2R compared with those with known primary $(p=0.04)$. 
immune tumor microenvironment, antigen presentation and cytotoxic and helper $\mathrm{T}$ cell activity. ${ }^{18}$ Furthermore, immune-related gene expression profiles were found to be predictive of response following PD-1 blockade therapy in patients with advanced NSCLC, head and neck squamous cell carcinoma, and melanoma. ${ }^{23}$ This is in addition to a $\mathrm{T}$ cell-inflamed gene expression profile tested in melanoma and nine other malignancies that is currently being evaluated in clinical trials testing pembrolizumab. ${ }^{24}$

Antigen presentation by MHC proteins is essential for adaptive immunity. We were interested in exploring MHC-I and MHC-II gene expression in unknown primary tumors compared with known primaries and used recently reported MHC-I and MHC-II scores in our analysis. ${ }^{15}$ MUP tumors had significantly higher MHC-I and MHC-II scores further supporting enhanced immunogenicity in MUP and a higher likelihood of response to immune checkpoint blockade. ${ }^{25}$ Overall, our findings and related literature support the hypothesis that interactive immune-related pathways related to tumor biology and host immunology may explain the improved prognosis in patients with MUP.

Although we tested a broad panel of candidate circulating and soluble biomarkers, only IL-2R was found to be significantly higher in MUP patients. This could be explained by the smaller sample size included in this analysis ( $\mathrm{N}=321 ; 66$ unknown and 255 known primary). These findings support the hypothesis that MUP patients are more likely to have immunogenic tumor microenvironments, and evidence of peripheral proinflammatory immune responses that drive immune resistance to melanoma and make MUP more susceptible to adjuvant immune checkpoint blockade interventions. Unknown primary is typically attributed to the phenomenon of complete regression at the primary melanoma site. ${ }^{4}$ Evidence of immunity to melanoma is known to be essential for disease control and improved prognosis in the adjuvant, neoadjuvant and inoperable disease settings. Melanoma spontaneous regression has been reported, suggesting a role for host immunity, that is also indirectly supported histologically by findings of tumor infiltrating lymphocytes in primary melanoma associated with tumor regression. ${ }^{26}$ Furthermore, lymphoid immune infiltrates within the tumor have been shown to be prognostic in primary melanoma, ${ }^{27}$ and melanoma metastatic to regional lymph nodes. ${ }^{28-30} \mathrm{~T}$ cell infiltrates within regional nodal metastasis were associated with response following neoadjuvant interferon- $\alpha$ and ipilimumab. ${ }^{17283132}$ The presence of these immune features in melanoma have been reported to be associated with benefit from systemic immunotherapy including cytokine therapy and immune checkpoint inhibitors. ${ }^{33}$ Therefore, a completely regressed primary in MUP may represent a consequence of prior host immune recognition and development of melanoma immune resistance that benefits from immunotherapeutic interventions. We propose that future adjuvant trials consider stratifying for MUP and we support the AJCC efforts in further investigating the prognostic value of MUP and its contributions to the melanoma staging system.

\section{Conclusions}

Unknown primary high-risk melanoma patients had a significantly better prognosis and showed evidence of significantly enhanced immune activation within the TME and the circulation, supporting the designation of unknown primary melanoma as a distinct favorable prognostic marker among patients with high-risk melanoma.

\section{Author affiliations}

${ }^{1}$ Cutaneous Oncology, Immunology, H. Lee Moffitt Cancer Center and Research Institute, Tampa, Florida, USA

${ }^{2}$ Biostatistics, Harvard Medical School, Boston, Massachusetts, USA

${ }^{3}$ Dana Farber Cancer Institute, Boston, Massachusetts, USA

${ }^{4}$ Biostatistics and Bioinformatics, H. Lee Moffitt Cancer Center and Research Institute, Tampa, Florida, USA

${ }^{5}$ Machine Learning, H. Lee Moffitt Cancer Center and Research Institute, Tampa, Florida, USA

${ }^{6}$ The Parker Institute for Cancer Immunotherapy, San Francisco, California, USA ${ }^{7}$ Microbiology, Immunology, University of California San Francisco, San Francisco, California, USA

${ }^{8}$ Pathology and Laboratory Medicine, Allegheny Cancer Institute, Allegheny Health Network, Pittsburgh, Pennsylvania, USA

${ }^{9}$ Immunology, Dermatology, University of Pittsburgh School of Medicine, Pittsburgh, Pennsylvania, USA

${ }^{10}$ UPMC Hillman Cancer Center, Pittsburgh, Pennsylvania, USA

${ }^{11}$ Pathology, University of Pittsburgh School of Medicine, Pittsburgh, PA, USA

${ }^{12}$ Immunology, H. Lee Moffitt Cancer Center and Research Institute, Tampa, Florida, USA

${ }^{13}$ Administration, Cutaneous Oncology, Immunology, H. Lee Moffitt Cancer Center and Research Institute, Tampa, Florida, USA

${ }^{14}$ Cancer Therapy Evaluation Program, National Cancer Institute, Rockville, Maryland, USA

${ }^{15}$ Cutaneous Oncology, H. Lee Moffitt Cancer Center and Research Institute, Tampa, Florida, USA

${ }^{16}$ Medicine, University of Pittsburgh School of Medicine, Pittsburgh, Pennsylvania, USA

Acknowledgements We thank the patients and their families and the investigators who participated in the E1609 study. The authors are also deeply indebted to Professor Uma N. M. Rao, MD who acted as the Pathology Co-Chair for the ECOGACRIN E1609 Phase III trial. Professor Rao passed away in 2020.

Contributors Conception and design: AAT, SJL, A-CT, IMEN, LHB and WAL. Provision of study material or patients: AAT, JMK, FSH, VKS, ECOG-ACRIN E1609 study investigators. Collection and assembly of data: AAT, JMK, SJL, FSH, VKS, ECOG-ACRIN E1609 study investigators. Data analysis: AAT, SJL, A-CT and IMEN. Data interpretation: AAT, SJL, A-CT, IMEN, LHB, WAL, WJS, FSH, JMK, VKS, ADK, JRC-G, PH and HS. Manuscript writing: all authors. Final approval of manuscript: all authors. Accountable for all aspects of the work: all authors. AAT is responsible for the overall content as guarantor.

Funding This study was coordinated by the ECOG-ACRIN Cancer Research Group (Peter J. O'Dwyer, MD and Mitchell D. Schnall, MD, PhD, Group Co-Chairs) and supported by the National Cancer Institute of the National Institutes of Health under the following award numbers: U10CA180820, U10180794, U10CA180821, U10CA180863, U10CA180888, UG1CA189859, UG1CA233163, UG1CA233180, UG1CA233184, UG1CA233196, UG1CA233234, UG1CA233237, UG1CA233270, UG1CA233331, UG1CA233373, UG1CA233320, UG1CA233337, Canadian Cancer Society \#704970. This study was also supported by Bristol-Myers Squibb. Biomarkers studies were supported under University of Pittsburgh Skin Spore award number P50CA12197310 (AAT, WJS, LHB and JMK). Support for Shared Resources was provided by Cancer Center Support Grant (CCSG) CA076292 to H. Lee Moffitt Cancer Center.

Disclaimer The content is solely the responsibility of the authors and does not necessarily represent the official views of the National Institutes of Health. Mention 
of trade names, commercial products, or organizations does not imply endorsement by the US government.

Competing interests AAT reports grants from National Cancer Institute, National Institute of Health, ECOG-ACRIN, grants from Bristol Myers Squibb, during the conduct of the study; grants from Bristol Myers Squibb, personal fees from Bristol Myers Squibb, grants from Merck, personal fees from Merck, personal fees from Novartis, personal fees from Genentech- Roche, grants from Genentech-Roche, personal fees from Array Biopharma, grants from Incyte, personal fees from Incyte, personal fees from NEWLINK Genetics, personal fees from HUYA, personal fees from BioNTech, grants from Prometheus, personal fees from Prometheus, personal fees from Immunocore, grants from Greenpeptide, grants from Amgen, grants from Clinigen, personal fees from Clinigen, personal fees from Partners Therapeutics, personal fees and grants from Regeneron, personal fees and grants from Sanofi-Genzyme outside the submitted work. SJL has nothing to disclose. A-CT reports has nothing to disclose. IMEN is a deputy editor for Medical Physics and reports relationship with Scientific Advisory Endectra, LLC. FSH reports clinical trial support from Eastern Cooperative oncology Group, during the conduct of the study; grants, personal fees and other from Bristol-Myers Squibb, personal fees from Merck, personal fees from EMD Serono, grants and personal fees from Novartis, personal fees from Takeda, personal fees from Surface, personal fees from Genentech/Roche, personal fees from Compass Therapeutics, personal fees from Apricity, personal fees from Bayer, personal fees from Aduro, personal fees from Partners Therapeutics, personal fees from Sanofi, personal fees from Pfizer, personal fees from Pionyr, from 7 Hills Pharma, personal fees from Verastem, other from Torque, personal fees from Rheos, outside the submitted work; in addition, FSH has a patent Methods for Treating MICA-Related Disorders (\#20100111973) with royalties paid, a patent Tumor antigens and uses thereof (\#7250291) issued, a patent Angiopoiten-2 Biomarkers Predictive of Anti-immune checkpoint response (\#20170248603) pending, a patent Compositions and Methods for Identification, Assessment, Prevention, and Treatment of Melanoma using PD-L1 Isoforms (\#20160340407) pending, a patent Therapeutic peptides (\#20160046716) pending, a patent Therapeutic Peptides (\#20140004112) pending, a patent Therapeutic Peptides (\#20170022275) pending, a patent Therapeutic Peptides (\#20170008962) pending, a patent THERAPEUTIC PEPTIDES Therapeutic Peptides Patent number: 9402905 issued, and a patent METHODS OF USING PEMBROLIZUMAB AND TREBANANIB pending. LHB declares the following unrelated advisory activities: StemImmune/Calidi Scientific and Medical Advisory Board, April 6, 2017-present; Western Oncolytics, Scientific Advisory Board, 2018-present; Torque Therapeutics, Scientific Advisory Board, 2018-2020; Khloris, Scientific Advisory Board, 2019-present; Pyxis, Scientific Advisory Board, 2019-present; Cytomix, Scientific Advisory Board, 2019-present; Vir, Scientific Advisory Board meeting, Feb. 2020; DCprime, Scientific Advisory Board meeting, Nov. 2020; RAPT, Scientific Advisory Board, 2020-present; Takeda, Scientific Advisor, 2020-present; EnaraBio scientific advisor, Feb. 2021. WAL has nothing to disclose. WJS has nothing to disclose. ADK has nothing to disclose. JRC-G has stock options with Compass Therapeutics, Anixa Biosciences and Alloy Therapeutics, receives honorarium from Anixa Biosciences, Alloy Therapeutics and Leidos, and has sponsored research with Anixa Biosciences. $\mathrm{PH}$ reports consulting fees consulting fees from Dragonfly and Immatics. HS has nothing to disclose. VKS reports personal fees from Merck, Bristol-Myers Squibb, Novartis, Array, Polynoma, Pfizer, and Regeneron, outside the submitted work. JMK reports grants and personal fees from Amgen, Bristol Myers Squibb, Castle Biosciences, Checkmate Pharmaceuticals, Immvira Pharma Co., Immunocore, lovance Biotherapeutics, Lion Biotechnologies, Merck, Novartis Pharmaceuticals, Schering-Plough, personal fees Ankyra Therapeutics, Axio Research/Instil Bio, Becker Pharmaceutical Consulting, DermTech, Elsevier, Fenix Group International, Harbour BioMed, Intellisphere/Cancer Network, IQVIA, Istari Oncology, Millennium Pharmaceutical/Takeda Pharmaceutical, Natera, OncoCyte, OncoSec, Pfizer, Replimune, Scopus BioPharma, SR One Captital Management, outside the submitted work.

\section{Patient consent for publication Not applicable.}

Ethics approval The study protocol was approved by the institutional review board of each participating institution and conducted in accordance with Good Clinical Practice guidelines as defined by the International Conference on Harmonization. All patients provided an IRB-approved written informed consent. This study was monitored by the ECOG-ACRIN Data Safety Monitoring Committee and the $\mathrm{NCl}$.

\section{Provenance and peer review Not commissioned; externally peer reviewed.}

Data availability statement Data are available on reasonable request. The data sets generated, analyzed and reported in the present paper will be made available in the NCTN/NCORP Data Archive (https://nctn-data-archive.nci.nih.gov).
Supplemental material This content has been supplied by the author(s). It has not been vetted by BMJ Publishing Group Limited (BMJ) and may not have been peer-reviewed. Any opinions or recommendations discussed are solely those of the author(s) and are not endorsed by BMJ. BMJ disclaims all liability and responsibility arising from any reliance placed on the content. Where the content includes any translated material, BMJ does not warrant the accuracy and reliability of the translations (including but not limited to local regulations, clinical guidelines, terminology, drug names and drug dosages), and is not responsible for any error and/or omissions arising from translation and adaptation or otherwise.

Open access This is an open access article distributed in accordance with the Creative Commons Attribution Non Commercial (CC BY-NC 4.0) license, which permits others to distribute, remix, adapt, build upon this work non-commercially, and license their derivative works on different terms, provided the original work is properly cited, appropriate credit is given, any changes made indicated, and the use is non-commercial. See http://creativecommons.org/licenses/by-nc/4.0/.

\section{ORCID iDs}

Ahmad A Tarhini http://orcid.org/0000-0002-3193-9702

Lisa H Butterfield http://orcid.org/0000-0002-3439-9844

Walter J Storkus http://orcid.org/0000-0001-8961-4444

\section{REFERENCES}

1 Siegel RL, Miller KD, Fuchs HE, et al. Cancer statistics, 2021. CA Cancer J Clin 2021;71:7-33.

2 Tarhini A, Atzinger C, Gupte-Singh K, et al. Treatment patterns and outcomes for patients with unresectable stage III and metastatic melanoma in the USA. J Comp Eff Res 2019;8:461-73.

3 Tarhini A, Ghate S, lonescu-Ittu R, et al. Stage III melanoma incidence and impact of transitioning to the 8th AJCC staging system: a US population-based study. Future Oncol 2019;15:359-70.

4 Gershenwald JE, Scolyer RA, Hess KR, et al. Melanoma staging: evidence-based changes in the American Joint Committee on Cancer eighth edition cancer staging manual. CA Cancer J Clin 2017;67:472-92.

5 Cormier JN, Xing Y, Feng L, et al. Metastatic melanoma to lymph nodes in patients with unknown primary sites. Cancer 2006;106:2012-20.

6 Lee CC, Faries MB, Wanek LA, et al. Improved survival after lymphadenectomy for nodal metastasis from an unknown primary melanoma. J Clin Oncol 2008;26:535-41.

7 van der Ploeg APT, Haydu LE, Spillane AJ, et al. Melanoma patients with an unknown primary tumor site have a better outcome than those with a known primary following therapeutic lymph node dissection for macroscopic (clinically palpable) nodal disease. Ann Surg Oncol 2014;21:3108-16.

8 Tarhini AA, Lee SJ, Hodi FS, et al. Phase III study of adjuvant ipilimumab ( 3 or $10 \mathrm{mg} / \mathrm{kg}$ ) versus high-dose interferon alfa- $2 \mathrm{~b}$ for resected high-risk melanoma: North American Intergroup E1609. J Clin Oncol 2020;38:567-75.

9 Irizarry RA, Hobbs B, Collin F, et al. Exploration, normalization, and summaries of high density oligonucleotide array probe level data. Biostatistics 2003;4:249-64.

10 Subramanian A, Tamayo P, Mootha VK, et al. Gene set enrichment analysis: a knowledge-based approach for interpreting genome-wide expression profiles. Proc Natl Acad Sci U S A 2005;102:15545-50.

11 Liberzon A, Subramanian A, Pinchback R, et al. Molecular signatures database (MSigDB) 3.0. Bioinformatics 2011;27:1739-40.

12 Newman AM, Liu CL, Green MR, et al. Robust enumeration of cell subsets from tissue expression profiles. Nat Methods 2015;12:453-7.

13 Seiler M, Peng S, Agrawal AA, et al. Somatic mutational landscape of splicing factor genes and their functional consequences across 33 cancer types. Cell Rep 2018;23:282-96.

14 Xie M, Lee K, Lockhart JH, et al. TIMEx: tumor-immune microenvironment deconvolution web-portal for bulk transcriptomics using pan-cancer scRNA-seq signatures. Bioinformatics 2021:3681-3. doi:10.1093/bioinformatics/btab244

15 Liu D, Lin J-R, Robitschek EJ, et al. Evolution of delayed resistance to immunotherapy in a melanoma responder. Nat Med 2021;27:985-92.

16 Butterfield LH, Potter DM, Kirkwood JM. Multiplex serum biomarker assessments: technical and biostatistical issues. $J$ Trans/ Med 2011;9:173.

17 Tarhini AA, Edington H, Butterfield LH, et al. Immune monitoring of the circulation and the tumor microenvironment in patients with regionally advanced melanoma receiving neoadjuvant ipilimumab. PLoS One 2014;9:e87705. 
18 Tarhini AA, Lin Y, Lin H-M, et al. Expression profiles of immunerelated genes are associated with neoadjuvant ipilimumab clinical benefit. Oncoimmunology 2017;6:e1231291.

19 Prens SP, van der Ploeg APT, van Akkooi ACJ, et al. Outcome after therapeutic lymph node dissection in patients with unknown primary melanoma site. Ann Surg Oncol 2011;18:3586-92.

20 Cormier JN, Xing Y, Feng L, et al. Metastatic melanoma to lymph nodes in patients with unknown primary sites. Cancer 2006;106:2012-20.

21 Gos A, Jurkowska M, van Akkooi A, et al. Molecular characterization and patient outcome of melanoma nodal metastases and an unknown primary site. Ann Surg Oncol 2014;21:4317-23.

22 Kanehisa M, Goto S, Furumichi M, et al. KEGG for representation and analysis of molecular networks involving diseases and drugs. Nucleic Acids Res 2010;38:D355-60.

23 Prat A, Navarro A, Paré L, et al. Immune-related gene expression profiling after PD-1 blockade in non-small cell lung carcinoma, head and neck squamous cell carcinoma, and melanoma. Cancer Res 2017;77:3540-50.

24 Ayers M, Lunceford J, Nebozhyn M, et al. IFN- $\gamma$-related mRNA profile predicts clinical response to PD-1 blockade. J Clin Invest 2017;127:2930-40.

25 Shklovskaya E, Lee JH, Lim SY, et al. Tumor MHC expression guides first-line immunotherapy selection in melanoma. Cancers 2020;12 doi:10.3390/cancers 12113374

26 Spontaneous regression of malignant melanoma: a review of the literature on incidence, clinical features, and possible mechanisms. Natl Cancer Inst Monogr 1976;44:67-77.
27 Clemente CG, Minm MC, Bufalino R, et al. Prognostic value of tumor infiltrating lymphocytes in the vertical growth phase of primary cutaneous melanoma. Cancer 1996;77:1303-10.

28 Mihm MC, Clemente CG, Cascinelli N. Tumor infiltrating lymphocytes in lymph node melanoma metastases: a histopathologic prognostic indicator and an expression of local immune response. Lab Invest 1996;74:43-7.

29 Erdag G, Schaefer JT, Smolkin ME, et al. Immunotype and immunohistologic characteristics of tumor-infiltrating immune cells are associated with clinical outcome in metastatic melanoma. Cancer Res 2012;72:1070-80.

30 Bogunovic D, O'Neill DW, Belitskaya-Levy I, et al. Immune profile and mitotic index of metastatic melanoma lesions enhance clinical staging in predicting patient survival. Proc Natl Acad Sci U S A 2009;106:20429-34.

31 Håkansson A, Gustafsson B, Krysander L, et al. Tumour-infiltrating lymphocytes in metastatic malignant melanoma and response to interferon alpha treatment. Br J Cancer 1996;74:670-6.

32 Moschos SJ, Edington HD, Land SR, et al. Neoadjuvant treatment of regional stage IIIB melanoma with high-dose interferon alfa-2b induces objective tumor regression in association with modulation of tumor infiltrating host cellular immune responses. J Clin Oncol 2006;24:3164-71.

33 Tarhini A, Kudchadkar RR. Predictive and on-treatment monitoring biomarkers in advanced melanoma: moving toward personalized medicine. Cancer Treat Rev 2018;71:8-18. 